\title{
Torque method for the theoretical determination of magnetocrystalline anisotropy
}

\author{
Xindong Wang \\ Department of Physics and Astronomy, Northwestern University, Evanston, Illinois 60208-3112 \\ Ruqian Wu \\ Department of Physics, California State University at Northridge, Northridge, California 91330-8268 \\ Ding-sheng Wang \\ Institute of Physics, Academia Sinica, Beijing 100080, People's Republic of China
}

A. J. Freeman

Department of Physics and Astronomy, Northwestern University, Evanston, Illinois 60208-3112

(Received 28 July 1995; revised manuscript received 12 February 1996)

\begin{abstract}
We propose a torque method for the theoretical determination of the magnetocrystalline anisotropy (MCA) energy for systems with uniaxial symmetry. While the dependence of the total energy on the angle between the magnetization and the normal axis $(\theta)$ can be expressed as $E(\theta)=E_{0}+K_{2} \sin ^{2}(\theta)+K_{4} \sin ^{4}(\theta)$, we show that the MCA energy [defined as $E_{\mathrm{MCA}}=E\left(\theta=90^{\circ}\right)-E\left(\theta=0^{\circ}\right)=K_{2}+K_{4}$ ] can be easily evaluated through the expectation value of the angular derivative of the spin-orbit coupling Hamiltonian (torque) at an angle of $\theta=45^{\circ}$. Unlike other procedures, the proposed method is independent of the validity of the MCA force theorem, or of the absolute accuracy of two total energy calculations. Calculated MCA energies for the free Fe monolayer with different lattice constants are analyzed and compared with results of other $a b$ initio calculations, especially those obtained with our previously reported state tracking method. [S0163-1829(96)02926-8]
\end{abstract}

Recent developments in magnetic thin films and overlayers that show perpendicular magnetic anisotropy ${ }^{1}$ with their tremendous practical implications for high-density magnetooptical storage media, have stimulated $a b$ initio theoretical determinations of the magnetocrystalline anisotropy energy (MAE). ${ }^{2-12}$ Unfortunately, despite the great advances in local-spin-density electronic structure theory and computational power in the past decades, the accurate determination of MAE still remains difficult and computationally demanding. In the traditional approach, the value of MAE is calculated through comparing the total energies of a given system for two different magnetization orientations (in-plane and perpendicular directions for surface-interface systems). Since the spin-orbit coupling (SOC) is very weak in $3 d$ transition metals, the so-called magnetocrystalline anisotropy (MCA) force theorem ${ }^{6}$ is usually adopted and the MAE is calculated by merely comparing the band energies between the two magnetic orientations. (We call them direct approaches.) The main difficulty associated with the direct approaches concerns the numerical stability of calculating a very small difference of two large numbers. In fact, in order to eliminate numerical fluctuations, ${ }^{3-7,9}$ extremely fine sampling meshes are required for the $k$-space integrations.

In this paper, we propose a torque method for the determination of MCA. The main advantage that this method offers is that one only needs to calculate MAE at one particular magnetic orientation and to do the $k$-space integration with the single Fermi surface at this orientation. In the following, we first present the method, then give results for free standing Fe monolayers as a test case, and compare them with the results of other methods. ${ }^{3-5,9}$
To demonstrate the idea, let us recall that the total energy of an uniaxial system can be well approximated in the form

$$
E(\theta)=E_{o}+K_{2} \sin ^{2}(\theta)+K_{4} \sin ^{4}(\theta),
$$

where $\theta$ is the angle between the magnetization and the normal axis. If we define the torque, $T(\theta)$, as the angular derivative of the total energy, i.e.,

$$
T(\theta) \equiv \frac{d E(\theta)}{d \theta}=K_{2} \sin (2 \theta)+2 K_{4} \sin (2 \theta) \sin ^{2}(\theta),
$$

it is easy to show that

$$
\begin{aligned}
M A E & \equiv E\left(\theta=90^{\circ}\right)-\Delta E\left(\theta=0^{\circ}\right) \\
& =K_{2}+K_{4}=T\left(\theta=45^{\circ}\right) .
\end{aligned}
$$

As usual, a positive MAE means a perpendicular magnetic anisotropy. Clearly, the MAE can be evaluated very efficiently through calculating the torque at $\theta=45^{\circ}$ even when $K_{4}$ is not negligible.

We next come to the question of how to calculate the torque. Within the framework of local-spin-density theory, including SOC, the total energy of the system is ${ }^{6}$

$$
E\left[\rho_{\sigma}(\theta) ; \theta\right]=\sum_{\text {occ. }} \epsilon\left[\rho_{\sigma}(\theta) ; \theta\right]+E^{\prime}\left[\rho_{\sigma}(\theta)\right]
$$

where we have separated the total energy into the KohnSham band energy and the remaining double counting terms, $E^{\prime}$. Note that the total energy depends on $\theta$ both explicitly 


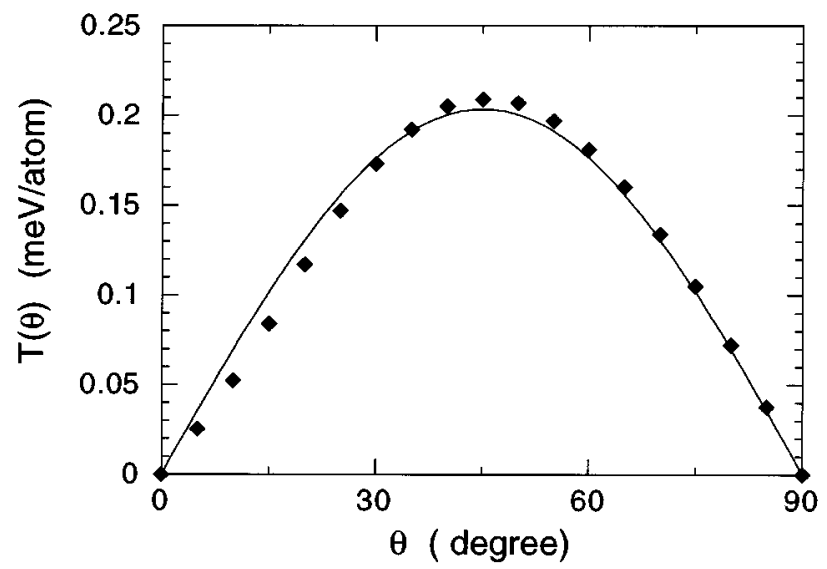

FIG. 1. Calculated torque vs the angle $\theta$ for an Fe monolayer ( $a=4.83$ a.u.). The diamonds are the calculated $T(\theta)$, and the solid line is the fitted $0.202 \sin (2 \theta)$ curve.

(through the SOC Hamiltonian) and implicitly (through the angular dependence of the charge and spin densities). These two sources of the dependence of the total energy on $\theta$ are not to be confused. To calculate the torque at a certain angle $\theta$, we can write

$$
\begin{aligned}
E\left[\rho_{\sigma}(\theta+\delta \theta) ; \theta+\delta \theta\right]-E\left[\rho_{\sigma}(\theta) ; \theta\right] & \\
= & \left\{E\left[\rho_{\sigma}(\theta+\delta \theta) ; \theta+\delta \theta\right]-E\left[\rho_{\sigma}(\theta) ; \theta+\delta \theta\right]\right\} \\
& +\left\{E\left[\rho_{\sigma}(\theta) ; \theta+\delta \theta\right]-E\left[\rho_{\sigma}(\theta) ; \theta\right]\right\} \\
= & O\left[\delta \theta^{2}\right] \quad+\sum_{\text {occ. }} \epsilon\left[\rho_{\sigma}(\theta) ; \theta+\delta \theta\right]-\sum_{\text {occ. }} \epsilon\left[\rho_{\sigma}(\theta) ; \theta\right] \\
= & \frac{1}{\Omega_{\mathrm{BZ}}} \int_{V_{F}} \delta \epsilon(\mathbf{k}) d \mathbf{k}+\frac{1}{\Omega_{\mathrm{BZ}}} \int_{\delta V_{F}} \epsilon(\mathbf{k}) d \mathbf{k} \\
= & \frac{1}{\Omega_{\mathrm{BZ}}} \int_{V_{F}} \delta \epsilon(\mathbf{k}) d \mathbf{k}+\frac{\epsilon_{F}}{\Omega_{\mathrm{BZ}}} \int_{\delta V_{F}} d \mathbf{k} \\
= & \frac{1}{\Omega_{\mathrm{BZ}}} \int_{V_{F}} \delta \epsilon(\mathbf{k}) d \mathbf{k},
\end{aligned}
$$

where $\Omega_{\mathrm{BZ}}$ is the volume of the Brillouin zone and we have made use of the stationary property of the total energy with respect to variations in charge-spin density (but not in $\theta$ ). Also in obtaining the last equality in Eq. (5), we have used the fact that the Fermi volume should remain constant.

Having obtained Eq. (5), we can apply the FeynmanHellman theorem to obtain the torque as

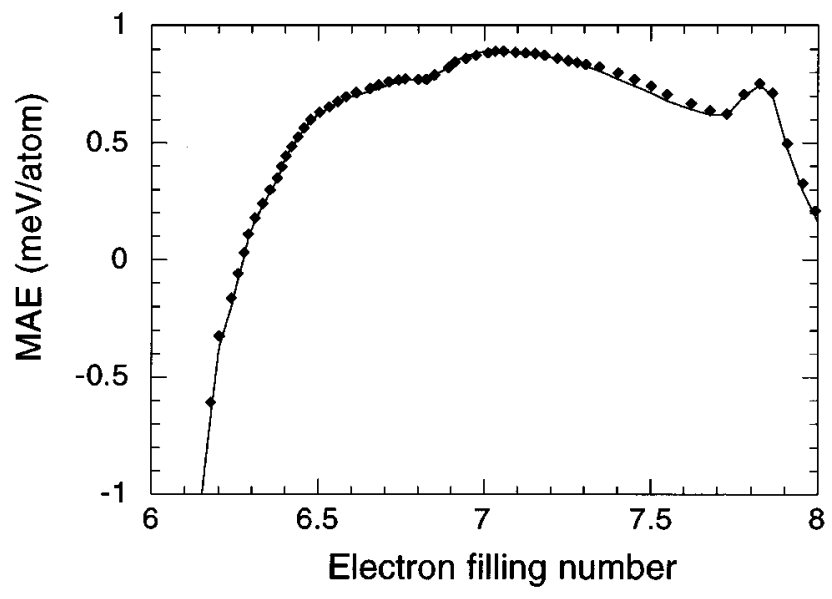

FIG. 2. MAE vs band filling for an Fe monolayer ( $a=4.83$ a.u.). The diamonds are the results obtained by the linear interpolation method in $k$-space integration, and the line is by the quadratic interpolation method. Convergence of the $k$-space integration may be assumed since the two agree to better than $0.05 \mathrm{meV}$.

$$
T(\theta)=\sum_{\text {occ. }}\left\langle\psi_{i, \mathbf{k}}^{\text {so }}\left|\frac{\partial H_{\text {so }}}{\partial \theta}\right| \psi_{i, \mathbf{k}}^{\text {so }}\right\rangle,
$$

where the spin-orbit coupling Hamiltonian is

$$
H_{\mathrm{so}}=\sum_{i} \xi\left(r_{i}\right) \hat{\boldsymbol{l}}_{i} \cdot \hat{\mathbf{s}}
$$

and the index $i$ refers to atoms and $\hat{l}_{i}=-i \mathbf{r}_{i} \times \nabla$. If we let the magnetization direction be $\mathbf{n}(\theta, \phi)$, we can write the spinorbit coupling term as

$$
\begin{aligned}
\hat{l}_{i} \cdot \hat{\mathbf{s}}= & \hat{s}_{n}\left(\hat{l}_{z} \cos \theta+\frac{1}{2} \hat{l}_{+} e^{-i \phi} \sin \theta+\frac{1}{2} \hat{l}_{-} e^{i \phi} \sin \theta\right) \\
& +\frac{1}{2} \hat{s}_{+}\left(-\hat{l}_{z} \sin \theta-\hat{l}_{+} e^{-i \phi} \sin ^{2} \frac{\theta}{2}+\hat{l}_{-} e^{i \phi} \cos ^{2} \frac{\theta}{2}\right) \\
& +\frac{1}{2} \hat{s}_{-}\left(-\hat{l}_{z} \sin \theta+\hat{l}_{+} e^{-i \phi} \cos ^{2} \frac{\theta}{2}-\hat{l}_{-} e^{i \phi} \sin ^{2} \frac{\theta}{2}\right),
\end{aligned}
$$

from which the derivative with respect to $\theta$ is straightforward.

Note that here the Fermi energy and wave functions are determined with the spin-orbit coupling included and the results obtained so far do not rely upon the validity of the MCA force theorem. ${ }^{13}$ Calculations can be simplified, however, for situations where the MCA force theorem is valid. In such cases, one only needs the self-consistent scalar relativ-

TABLE I. Calculated Fe monolayer MAE (meV/atom) by different groups and/or methods (see text for description and comparisons).

\begin{tabular}{lcccc}
\hline \hline$a$ (a.u.) & Torque method & State tracking (Ref. 9) & LCAO (Refs. 3 and 4) & FLAPW (Ref. 5) \\
\hline 4.83 & 0.21 & 0.42 & 0.61 (Ref. 3) & \\
5.45 & -0.10 & 0.37 & 0.38 (Ref. 4) & $-0.033--0.043$ \\
\hline \hline
\end{tabular}


istic charge-spin density or potential and then performs one second-variational calculation with the SOC Hamiltonian invoked. This is the procedure adopted in the calculations presented below.

We used the full-potential linearized augmented plane wave (FLAPW) method with a slab geometry ${ }^{14}$ to get the self-consistent scalar relativistic charge-spin densities and potentials. With $55 k$ points in the $1 / 8$ irreducible BZ, selfconsistency for the scalar relativistic calculations is assumed when the rms difference between the input-output charge and spin densities become better than $10^{-4}$ e/a.u. ${ }^{3}$. To determine the MAE, we use triangular interpolation for $k$-space integrations. The number of $k$ points is assumed to be sufficient when the linear and quadratic interpolations give better than $0.05 \mathrm{meV}$ agreement. We found, for most cases, that a 20 by 20 division of the $\mathrm{BZ}$ is sufficient to assure convergence.

A good test whether our numerical results are stable enough against noise from the finite $k$ points used in the $k$-space integrations for torque is to calculate torques for different $\theta$. As indicated in Eq. (2), the torque versus $\theta$ should be a smooth function and in most cases, since $K_{4} \ll K_{2}$, we should expect a $\sin (2 \theta)$ behavior. Since we used the self-consistent scalar relativistic potential in our calculation, we can only be sure of the leading order contribution to the MAE, i.e., $K_{2}$ (Ref. 13) and no meaningful $K_{4}$ value can be obtained. To demonstrate that this is actually true, we show in Fig. 1 the $T(\theta)$ curve for an $\mathrm{Fe}$ monolayer with lattice constant 4.83 a.u. We see that well within our convergence criteria, the calculated points fit nicely to a $0.202 \sin (2 \theta)$ curve. Also shown in Fig. 2 for this same test system is the torque calculated at $45^{\circ}$ versus band filling where a rigid band picture is adopted. We see that the linear interpolation results (diamonds) agree everywhere with the quadratic interpolation (solid line) to better than $0.05 \mathrm{meV}$.

For Fe monolayers, there have been several published $a b$ initio results. ${ }^{3-5,9}$ Shown in Table I is a comparison between our results and previous attempts using different methods. ${ }^{3-5,9}$ Although the trend for MAE in going from smaller to larger lattice constant is the same among the different groups (i.e., as the lattice constant increases MAE becomes less positive), several comments are necessary for the apparent disagreement in the actual numbers. Gay and Richter $^{3,4}$ employed a traditional direct approach and used $14000 k$ points in the Brillouin zone to achieve $k$-space convergence. However, their linear combination of atomic orbitals (LCAO) band structure calculation method is less accurate than the FLAPW. An early FLAPW calculation done by Li et al. ${ }^{5}$ also used blind Fermi filling ${ }^{9}$ and the result is given within a certain range due to numerical uncertainties. Although the state tracking method used by Wang et al. ${ }^{9}$ was demonstrated to be very efficient in suppressing the fluctuations caused by blind Fermi filling, the small set of sampling $k$ points employed in the irreducible Brillouin zone (15k points for semirelativistic self-consistency and $66 k$ points for MAE integrations) also raised some uncertainties.

In fact, we found that with more than $210 k$ points, the state tracking approach gives almost the same MAE as does the present torque approach. This is reasonable because the contribution due to the slightly different Fermi surface in the two approaches, which to order $\xi^{2}$ can be explicitly expressed as $\left(E_{F} / \Omega_{\mathrm{BZ}}\right) \int_{\delta V_{F}} d \mathbf{k}$, is zero because the number of
TABLE II. Calculated MAE (meV/atom) for Fe monolayer systems with lattice constant 4.83 and 5.45 a.u. using the torque method and state tracking method. Convergence of $k$-space integration in both methods is made better than $0.05 \mathrm{meV} / \mathrm{atom}$.

\begin{tabular}{lcc}
\hline \hline$a$ (a.u.) & Torque method & State tracking \\
\hline 4.83 & 0.21 & 0.24 \\
5.45 & -0.10 & 0.00 \\
\hline \hline
\end{tabular}

electrons is fixed. In surface-interface systems, it is well known that the dominant contribution to MAE is of the order of $\xi^{2}$, and so as a practical method the state tracking approach is a very good approximation in determining the leading order contribution to the MAE for these systems. Indeed, when the MCA force theorem is to be applied with a limited number of $k$ points, the state tracking method is necessary to ensure that the change of charge and spin densities be of order $\xi^{2}$ and thus to suppress the Fermi surface fluctuations. ${ }^{9}$

Since for transition metals, contributions to the MAE from terms of higher order than $\xi^{2}$ was estimated to be $\sim 0.1 \mathrm{meV} /$ atom, ${ }^{9}$ different methods of determining the Fermi surface should agree within this range. Our wellconverged results using the torque method (where the Fermi surface is determined by Fermi-Dirac statistics) and using the state tracking method do confirm this conclusion. This is demonstrated in Table II where the results obtained by the torque and state tracking methods for $\mathrm{Fe}$ monolayers with lattice constants equal to 4.83 and 5.45 a.u. are shown. These numbers match the fcc $\mathrm{Cu}(100)$ and $\mathrm{Ag}(100)$ substrates' lattice constants, respectively.

The numerical advantage of the torque method is obvious even compared to the state tracking approach. First, one only has to deal with one magnetization direction and hence with only one Fermi surface. This is a very important feature especially when some heavy elements (e.g., Pt) are involved for which the spin-orbit coupling has to be treated selfconsistently. Since now the MAE can be obtained with only one angle, we can easily get rid of the numerical uncertainty inherent in the direct approaches whereas current total energy calculations can hardly achieve a precision of better than $1 \mathrm{meV}$. In addition, the MAE is evaluated as the expectation value of the angular derivative of the SOC Hamiltonian over all the occupied states. The contribution of the nearly degenerate states at $E_{F}$ (the so-called surface pair coupling) is thus effectively suppressed. In fact, we found that a combination of the state tracking and the torque approaches provide very stable results of the MAE even for complex systems such as $\mathrm{Co} / \mathrm{Cu}$ sandwich and superlattice systems. ${ }^{15}$ Very recently, this approach was also successfully applied for the determination of the tiny magnetostriction in bulk transition metals and thin films. ${ }^{16}$

We thank F. Y. Rao for help with some of the calculations. Conversations with V. P. Antropov and O. N. Myrasov on torque methods from the Green's function point of view were also helpful. This work was supported by the Office of Naval Research (Grant No. N00014-94-1-0030 and N0001495-1-0489) and a grant of computer time at the Arctic Region Supercomputing Center. 
${ }^{1}$ L.M. Falicov, D.T. Pierce, S.D. Bader, R. Gronsky, K.B. Hathaway, H.J. Hopster, D.N. Lambeth, S.P. Parkin, G. Prinz, M. Salamon, I.K. Schuller, and R.H. Victoria, J. Mater. Res. 5, 1299 (1990); S.D. Bader, Proc. IEEE 78, 909 (1990).

${ }^{2}$ H. Brooks, Phys. Rev. 58, 909 (1940); G.C. Fletcher, Proc. Phys. Soc. 78, 145 (1961); A.J. Bennett and B.R. Cooper, Phys. Rev. B 3, 1642 (1971); N. Mori, Y. Fukuda, and T. Ukai, J. Phys. Soc. Jpn. 37, 1263 (1974); P. Bruno, Phys. Rev. B 39, 865 (1989).

${ }^{3}$ J.G. Gay and R. Richter, Phys. Rev. Lett. 56, 2728 (1986).

${ }^{4}$ J.G. Gay and R. Richter, J. Appl. Phys. 61, 3362 (1987).

${ }^{5}$ C. Li, A.J. Freeman, H.J.F. Jansen, and C.L. Fu, Phys. Rev. B 42, 5433 (1990).

${ }^{6}$ G.H.O. Daalderrop, P.J. Kelly, and M.F.H. Schuurmans, Phys. Rev. B 41, 11919 (1990).

${ }^{7}$ G.H.O. Daalderrop, P.J. Kelly, and M.F.H. Schuurmans, Phys. Rev. B 42, 7270 (1990); G.H.O. Daalderrop, P.J. Kelly, and F.J.A. den Broeder, Phys. Rev. Lett. 68, 682 (1992).

${ }^{8}$ P. Strange, J.B. Stauton, B.L. Gyorffy, and H. Ebert, Physica B 172, 51 (1991).
${ }^{9}$ D.S. Wang, R. Wu, and A.J. Freeman, Phys. Rev. Lett. 70, 869 (1993).

${ }^{10}$ D.S. Wang, R. Wu, and A.J. Freeman, J. Appl. Phys. 73, 6745 (1993); Phys. Rev. B 47, 14932 (1993); J. Magn. Magn. Mater. 129, 237 (1994); Phys. Rev. B 48, 15886 (1993).

${ }^{11}$ R.H. Victora and J.M. MacLaren, Phys. Rev. B 47, 11583 (1993).

${ }^{12}$ I.V. Solovyev, Fiz. Met. Metall. 75, 15 (1993); 76, 102 (1993); I.V. Solovyev, P.H. Dederichs, and I. Mertig (unpublished).

${ }^{13}$ X.D. Wang, D.S. Wang, R. Wu, and A.J. Freeman (unpublished).

${ }^{14}$ H. Krakauer, M. Posternak, and A.J. Freeman, Phys. Rev. Lett. 41, 1072 (1978); E. Wimmer, H. Krakauer, and A.J. Freeman, Phys. Rev. B 24, 864 (1981); M. Weinert, E. Wimmer, and A.J. Freeman, ibid. 26, 4571 (1982); E. Wimmer, H. Krakauer, and A.J. Freeman, in Advances in Electronics and Electron Physics, edited by P.W. Hawkes (Academic Press, Orlando, 1985), Vol. 65 , p. 357.

${ }^{15}$ L.P. Zhong, M.Y. Kim, X.D. Wang, A.J. Freeman, and D.S. Wang, Phys. Rev. B 53, 9770 (1996).

${ }^{16}$ R.Q. Wu and A.J. Freeman (unpublished). 